\title{
Coercivity analysis in sputtered Sm-Co thin films
}

\author{
C. Prados and A. Hernando \\ Instituto de Magnetismo Aplicado, P.O. Box 155, 28230 Las Rozas, Madrid, Spain \\ G. C. Hadjipanayis \\ University of Delaware, Department of Physics and Astronomy, Newark, Delaware 19716 \\ J. M. González ${ }^{\mathrm{a})}$ \\ Instituto de Ciencia de Materiales de Madrid-CSIC, Cantoblanco, 28049 Madrid, Spain
}

\begin{abstract}
Hard nanocrystalline $\mathrm{Sm}-\mathrm{Co}$ thin films having a thickness of $500 \mathrm{~nm}$ have been prepared by heat-treating magnetron sputtered amorphous samples deposited over a $300 \mathrm{~nm} \mathrm{Cr}$ buffer layer onto room temperature water cooled $\mathrm{Si}$ substrates. The thermal treatments resulted in the nanocrystallization of the precursor films and in the development of room temperature coercivities covering a range going from a few $\mathrm{kOe}$, measured in the samples treated at low temperature, up to some tens of kOe, observed in the case of the films annealed at high temperatures (the room temperature coercivity of the sample treated at $825 \mathrm{~K}$ was $26 \mathrm{kOe}$ ). Our analysis of the influence of the thermal treatments on the particularities of the reversal process was based on the measurement of the temperature dependence of the coercive force. It was carried out in the framework of the micromagnetic model, which allowed us to evaluate two parameters accounting for the local anisotropy reduction and the magnitude of the local dipolar fields, respectively. Our results show that the high temperature treatments result in a better degree of crystallization (lower local anisotropy deterioration). That crystallinity improvement is associated to an increase of the local dipolar interactions. (C) 1999 American Institute of Physics. [S0021-8979(99)68808-9]
\end{abstract}

\section{INTRODUCTION}

Thin films of hard magnetic materials, such as the rareearth transition metal alloys, have stimulated an active research aiming at their application in different devices and in magnetic recording media. SmCo films with Cr underlayer, prepared under different conditions, exhibiting high magnetic coercivity, small grain size, and high uniaxial anisotropy have shown a good potential for high density recording media. ${ }^{1-4}$ Recently, room temperature giant coercivity values have been reported in nanocrystalline $\mathrm{Sm}(\mathrm{Co}, \mathrm{Cu}, \mathrm{Ni}) / \mathrm{Cr}$ bilayers, in which the nanocrystalline structure was achieved by postannealing of the initially amorphous as-deposited films. ${ }^{5}$ The possibility of tuning the coercivity through the variation of the heat treatment parameters raises a question about the link between the reversal process and the structural and microstructural modifications induced by the anneals.

The characteristics of the magnetization reversal process of a hard magnetic film and, particularly, the magnitude of its coercive force depend on both the intrinsic and the extrinsic properties of the sample. The intrinsic properties (i.e., magnetocrystalline anisotropy, saturation magnetization, and exchange correlation length) are usually considered requisites for the development of the magnetic hardness and determine the choice of the composition of the main phase present in the film. Nevertheless, the actual coercivity value is ruled by a range of extrinsic features, including from the morphology (e.g., main phase grain shape and size) to the distribution of sites where the local values of the intrinsic properties depart from their bulk values (i.e., magnetic de-

\footnotetext{
${ }^{a)}$ Electronic mail: immgf4a@fresno.csic.es
}

fects). Those extrinsic features should be controlled during the deposition and treatment processes in order to get maximum coercivities. It is then crucial, in order to optimize the hard magnetic behavior, to try to clarify the relationships between the reversal process and all the mentioned extrinsic properties. The basic way of examining those relationships is the analysis of the temperature dependence of the coercive force. ${ }^{6}$ That analysis is based on the link, initially proposed by Brown ${ }^{7}$ and thoroughly developed by Kronmüller, ${ }^{6,8}$ between the coercivity, $H_{C}$, the anisotropy field, $H_{K}$, and the saturation magnetization, $M_{S}$ :

$$
H_{C}(T)=\alpha_{\varphi} \alpha_{K} H_{K}(T)-N_{\mathrm{eff}} M_{S}(T),
$$

where $\alpha_{\varphi}$ is given by the texture of the hard phase grains and $\alpha_{K}$ and $N_{\text {eff }}$ are fitting parameters depending on the local anisotropy and the stray fields, respectively. Despite its simplicity (it reduces the whole microstructure to only three parameters) this treatment (usually known as the micromagnetic model) has evidenced to be very useful to understand the behavior of permanent magnet samples. ${ }^{9}$

\section{EXPERIMENT}

$\mathrm{SmCo} / \mathrm{Cr}$ bilayers were deposited on $\mathrm{Si}$ substrates by means of a direct-current (dc) magnetron sputtering system without substrate heating. The base pressure was better than $10^{-7}$ Torr and $\mathrm{Ar}$ at a pressure of $5 \mathrm{mTorr}$ was used as discharge gas. The $\mathrm{Cr}$ underlayers were deposited from a pure (99.9) solid $\mathrm{Cr}$ target. A pressed-powder $\mathrm{SmCo}_{5}$ target was used to grow the SmCo layers. The deposition rates were $1.25 \AA / \mathrm{s}$ for $\mathrm{Cr}$ and $6 \AA / \mathrm{s}$ for $\mathrm{SmCo}$, and were calibrated by low angle $\mathrm{x}$-ray diffractometry (XRD). The thick- 


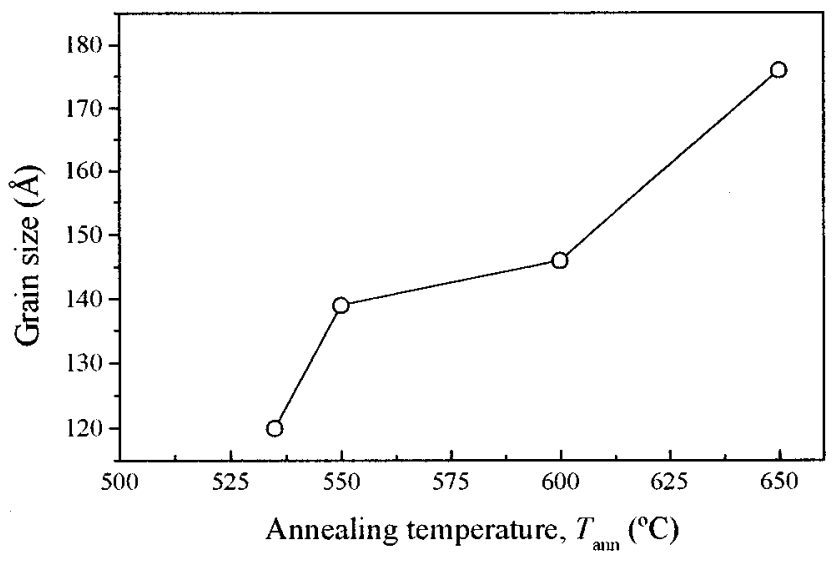

FIG. 1. Annealing temperature dependence of the average grain size.

ness of the $\mathrm{Cr}$ underlayer and the SmCo films were 300 and $500 \mathrm{~nm}$, respectively. A capping layer $(15 \mathrm{~nm}$ of $\mathrm{Cr})$ was deposited in order to protect the bilayer. Different pieces of the same as-deposited sample were heat treated for $30 \mathrm{~min}$ in vacuum $\left(10^{-5}\right.$ Torr $)$. The annealing temperatures, which ranged from 500 up to $600^{\circ} \mathrm{C}$, were used to designate the heat treatment. The structural properties of the annealed films were determined by XRD. The magnetic properties were measured by means of a superconducting quantum interference device (SQUID) magnetometer with a maximum field of $55 \mathrm{kOe}$ and in the temperature range from 5 up to $350 \mathrm{~K}$. The thermal evolution of the coercivity was determined as follows: (i) the bilayers were magnetized by applying the maximum available magnetic field at the maximum temperature; (ii) the samples were then field cooled down to the measurement temperature; (iii) increasing reversed fields were applied in order to demagnetize the sample.

\section{RESULTS AND DISCUSSION}

After the thermal treatments, the as-deposited amorphous films exhibited $\mathrm{SmCo}_{5}$ XRD reflections as well as a residual amorphous halo whose intensity decreased with the increase of the annealing temperature clearly indicating the

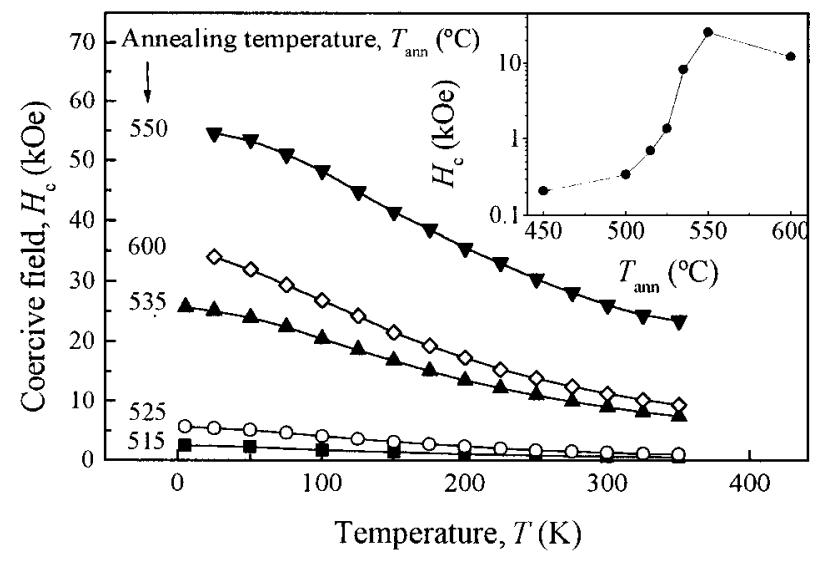

FIG. 2. Temperature dependence of the coercive force of films treated at different temperatures. The inset shows the annealing temperature dependence of the room temperature coercive force.

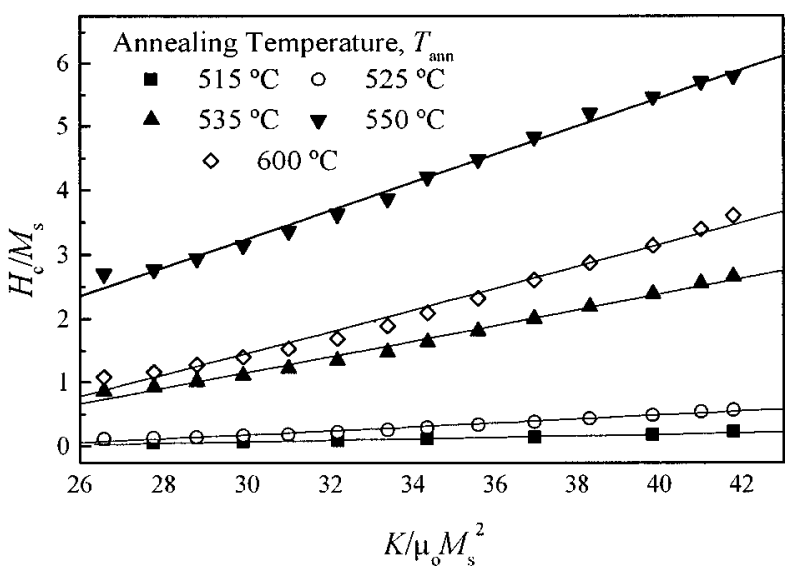

FIG. 3. Fit to Eq. (1) of the coercivity data.

attainment of a larger fraction of the crystallization process. Figure 1 presents the annealing temperature dependence of the average structural coherence length (grain size) as evaluated, by means of the Scherrer formula, from the measurement of the width of the (111) $\mathrm{SmCo}_{5}$ reflections. These data show the monotonous increase, from 12 up to $18 \mathrm{~nm}$, of the average grain size with the increase of the treatment temperature.

Our results for the temperature dependence of the saturation coercive force of the annealed films are presented in Fig. 2 (the inset shows the annealing temperature dependence of the room temperature coercivity). As it is apparent from the figure, in all the films the coercive force monotonously increased with the decrease of the temperature. The data in Fig. 2 were analyzed in the framework of the micromagnetic model by considering the temperature dependencies of the saturation magnetization and the anisotropy field of $\mathrm{SmCo}_{5}{ }^{10}$ and $\alpha_{\varphi}=0.5$ ( $\alpha_{\varphi}$ was evaluated following ${ }^{6}$ and considering nonexchange coupled, isotropically distributed grains). The corresponding fits are presented in Fig. 3 and gives evidence that expression (1) adequately describes the observed coercivity behavior.

Figure 4 presents the annealing temperature dependence of the $\alpha_{K}$ and $N_{\text {eff }}$ parameters. From this figure it is clear that

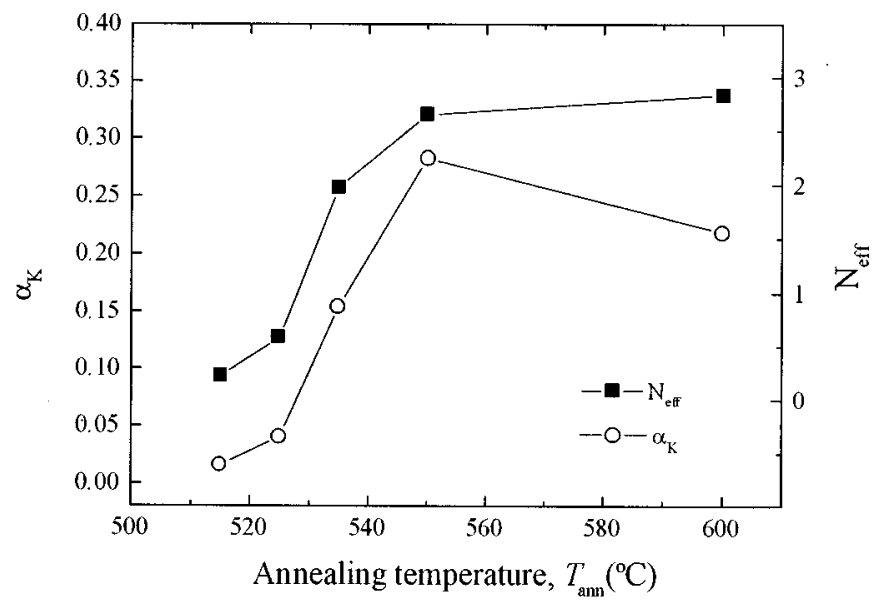

FIG. 4. Annealing temperature dependence of $\alpha_{K}$ and $N_{\text {eff }}$. 


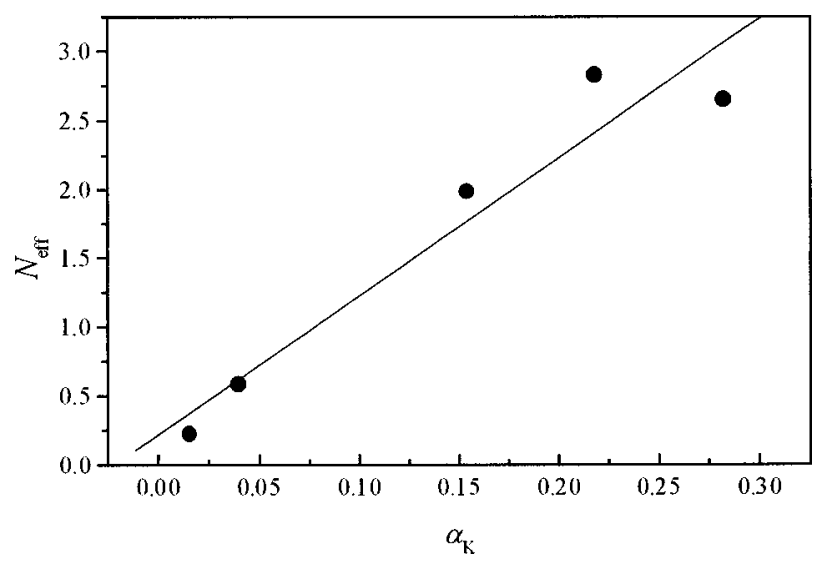

FIG. 5. $N_{\text {eff }}$ dependence on $\alpha_{K}$ (data corresponding to samples annealed at different temperatures).

the development of high coercivities (samples treated above $525^{\circ} \mathrm{C}$ ) is linked to the achievement of the highest $\alpha_{K}$ values, which is understood in the sense that the higher the $H_{c}$ value, the better the crystallinity of the SmCo grains. ${ }^{6}$ Also, we should point out that the obtained $N_{\text {eff }}$ values (in some cases clearly larger than 1) increase with the increase of the annealing temperature which could be ascribed, in close relationship to the annealing temperature evolution of $\alpha_{K}$, to the development of polyhedral grains having sharp edges and corners.
It is also interesting to point out that $N_{\text {eff }}$ depends, in a close to linear way, on $\alpha_{K}$ (see Fig. 5) which suggests that the magnetic pole distribution, at the place from where the magnetization reversal proceeds, is strongly influenced by the degree of crystallinity of the hard phase (the better the crystallinity the sharper the grain edges and corners). Finally, we propose that the optimum annealing conditions reflect the compromise between small local anisotropy deterioration (large $\alpha_{K}$ values) and a relatively small magnitude of the local stray field (linked to small $N_{\text {eff }}$ values). That compromise results in our samples in optimum coercivity in the case of the $550{ }^{\circ} \mathrm{C}$ annealed sample.

${ }^{1}$ E. M. T. Velu and D. N. Lambeth, J. Appl. Phys. 69, 5175 (1991).

${ }^{2}$ Y. Okomura, H. Fujimori, O. Suzuki, N. Hosoya, X. B. Yang, and H. Morita, IEEE Trans. Magn. 30, 4038 (1994).

${ }^{3}$ S. S. Malhotra, Y. Liu, Z. S. Shan, S. H. Liou, D. C. Stafford, and D. J. Sellmyer, J. Appl. Phys. 79, 5958 (1996).

${ }^{4}$ S. Takei, S. Shomura, A. Morisako, and M. Matsumoto, J. Appl. Phys. 81, 4674 (1997).

${ }^{5}$ C. Prados and G. C. Hadjipanayis, J. Appl. Phys. 83, 6253 (1998); C. Prados and G. C. Hadjipanayis, Appl. Phys. Lett. (in press).

${ }^{6}$ H. Kronmüller, K.-D. Durst, and M. Sagawa, J. Magn. Magn. Mater. 74, 291 (1988).

${ }^{7}$ W. F. Brown, Jr., Rev. Mod. Phys. 17, 15 (1945).

${ }^{8}$ H. Kronmüller, in Supermagnets, Hard Magnetic Materials, edited by G. J. Long and F. Grandjean (Kluwer, The Netherlands, 1991), p. 461.

${ }^{9}$ H. Kronmüller, Phys. Status Solidi B 144, 385 (1987).

${ }^{10}$ H. Kirchmayr and B. Burzo, in "Landolt-Bornstein, New Series"' (III/ 19d2) (unpublished), p. 262 